\title{
Scattering of spinon excitations by potentials in the 1D Heisenberg model
}

\author{
A. Pavlis ${ }^{1,2}$ and X. Zotos ${ }^{1,2,3}$ \\ ${ }^{1}$ ITCP and CCQCN, Department of Physics, University of Crete, Herakleio, Greece \\ ${ }^{2}$ Foundation for Research and Technology - Hellas, 71110 Heraklion, Greece and \\ ${ }^{3}$ Leibniz Institute for Solid State and Materials Research IFW Dresden, 01171 Dresden, Germany
}

\begin{abstract}
By a semi-analytical Bethe ansatz method and a T-matrix approach we study the scattering of a spinon, the elementary quantum many-body topological excitation in the 1D Heisenberg model, by local and phonon potentials. In particular, we contrast the scattering of a spinon to that of a free spinless fermion in the XY model to highlight the effect of strong correlations. For the one spinon scattering in an odd-site chain, we find a regular behavior of the scattering coefficients. In contrast, in an even-site chain there is a transfer of transmission probability between the two spinon branches that grows exponentially with system size. We link the exponent of the exponential behavior to the dressed charge that characterizes the critical properties of the 1D Heisenberg model, an interplay of topological and critical properties. The aim of this study is a microscopic understanding of spinon scattering by impurities, barriers or phonons, modeled as prototype potentials, an input in the analysis of quantum spin transport experiments.
\end{abstract}

\section{INTRODUCTION}

The novel mode of thermal transport by magnetic excitations in quasi-one dimensional quantum magnets has been over the last few years the focus of extensive experimental [1] and theoretical studies [2-7]. It was promoted by the fortuitous coincidence of synthesis of excellent quality compounds very well described by prototype integrable spin chain models and the proposal of unconventional -ballistic- spin and thermal transport in these systems [2]. Of course the purely ballistic thermal transport predicted by theory is not observed in thermal conduction experiments as the, albeit very high, thermal conductivity is limited by the scattering of the magnetic excitations from impurities and phonons [1].

In parallel, in the field of spintronics (spin caloritronics) there is renewed interest in the transport of magnetization, with the (inverse) spin Hall and spin Seebeck effect employed for the generation and detection of spin currents $[8,9]$. So far mostly metallic, semiconducting and magnetically ordered (ferro, antiferro, ferri) magnetic materials have been studied. Only very recently the spin Seebeck effect was studied in the quasi-one dimensional quantum magnet $\mathrm{Sr}_{2} \mathrm{CuO}_{3}$ accurately described by a spin-1/2 Heisenberg chain [10].

Regarding quasi-one dimensional quantum magnets, a lot is known on their bulk thermodynamic [11] and magnetothermal transport properties [12-14]. The prototype model for these systems is the well studied 1D Heisenberg model that is analytically solvable by the Bethe ansatz (BA) method. The elementary excitations in this strongly correlated system are topological in nature - the spinons [15] - and most of thermodynamic and transport experiments are discussed in terms of these low energy excitations $[1,4,6]$.

In this work, we study the scattering of a spinon from local potentials aiming at a microscopic understanding of scattering processes by impurities, phonons and bar- riers, relevant to (far-out of equilibrium) quantum spin transport. At the moment, we do not address any particular experiment, we only present background work on the theoretical question, how does a quantum many-body topological excitation scatters from a potential ?. This question is also relevant in other systems with topological excitations of actual experimental and theoretical interest.

To this end, we first use a recently developed semianalytical Bethe ansatz method $[16,36]$ to evaluate scattering matrix elements by prototype potentials and then to evaluate scattering coefficients by a T-matrix method. We should emphasize that although it is an elementary exercise to evaluate the quantum mechanical scattering coefficients (reflection, transmission) of a free particle from a potential barrier, little is known on the scattering of a quantum many-body quasi-particle excitation even more so for a topological one. The Bethe ansatz solvable models offer exactly such a framework for the study of this fundamental problem.

\section{MODEL AND MATRIX ELEMENTS}

The XXZ anisotropic Heisenberg Hamiltonian for a chain of $N$ sites with periodic boundary conditions $S_{N+1}^{a}=S_{1}^{a}$ and in the presence of a local potential $V$ of strength $g$ is given by:

$$
\begin{aligned}
H & =\sum_{n=1}^{N} h_{n, n+1}+g V \\
h_{n, n+1} & =J\left(S_{n}^{x} S_{n+1}^{x}+S_{n}^{y} S_{n+1}^{y}+\Delta S_{n}^{z} S_{n+1}^{z}-h S_{n}^{z}\right),
\end{aligned}
$$

where $S_{n}^{a}=\frac{1}{2} \sigma_{n}^{a}, \sigma_{n}^{a}$ are Pauli spin operators with components $a=x, y, z$ at site $n, \mathrm{~h}$ is the magnetic field and the anisotropy parameter $\Delta$ is typically parametrized as $\Delta=\cos \gamma$. In the following we will focus in the easy-plane 


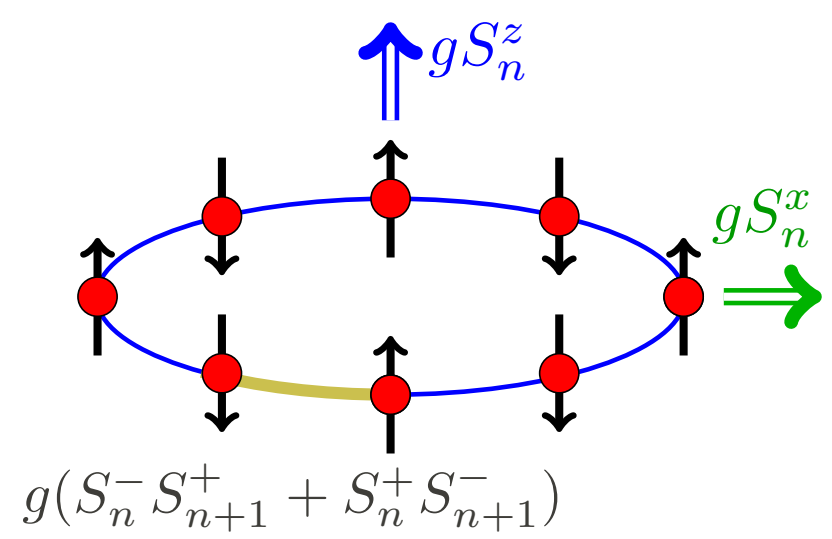

FIG. 1: Schematic figure of the spin chain and the type of potentials in consideration.

antiferromagnet, $0 \leq \Delta \leq 1$ and we will take $J=1$ as the unit of energy.

We study chains with odd as well as even number $N$ of spins. In odd chains, for each total $S^{z}= \pm 1 / 2$, the ground state is doubly degenerate containing one spinon with dispersion given by the one-branch $\varepsilon_{Q}=$ $v_{s}|\sin Q|, \quad 0<Q<\pi$. For even $\mathrm{N}$ the lowest excitations involve at least two spinons, the dispersion of each spinon given by $\varepsilon_{Q}=v_{s}|\sin Q|$ i.e. states of the Cloizeaux-Pearson spinon spectrum [15, 18]. We will study states belonging to the lowest energy branch of the $M=N / 2-1$ magnetization sector and obtained from the $S^{z}=1$ states by keeping the one spinon momentum fixed at zero and considering the dispersion of the second. In the spinon dispersion, $v_{s}=\frac{\pi}{2} \frac{\sin \gamma}{\gamma}$ and $Q$ is defined as the spinon momentum above the ground state. Normalized spinon states $|Q\rangle=|\{\lambda\}\rangle$ are determined from a specific set of Bethe roots $\left\{\lambda_{j}\right\}_{j=1}^{M}$, Supplemental Material [19] (see, also, reference [1] therein), and matrix elements between such states describe spinon scattering processes. Moreover we define the spinon group velocity as $u_{Q}=d \varepsilon_{Q} / d Q$.

In the following we first evaluate scattering matrix elements $|\mathcal{M}|^{2}=\left|\left\langle Q^{\prime}|V| Q\right\rangle\right|^{2}$ of a spinon from a state of momentum $Q$ to a state of momentum $Q^{\prime}$ on finite size lattices following $[16,19,36]$. We show in particular that they are strongly enhanced compared to those of single particle excitations leading to unusual scattering coefficients. The potentials we consider are schematically shown in Fig.1.

To start with we consider a one-site longitudinal potential $V=S_{n}^{z}$ at site $n$. The corresponding matrix element is given by [20],

$$
\left|\mathcal{M}_{q}^{z}(Q)\right|^{2}=|<Q+q| S_{q}^{z}|Q>|^{2}
$$

where $S_{n}^{z}=\frac{1}{\sqrt{N}} \sum_{q} e^{-i q n} S_{q}^{z}$. In the simple $\Delta=0$ case
- XY model - by a Jordan-Wigner transformation the spectrum corresponds to that of free spinless fermions, $\left|M_{q}^{z}\right|^{2}=1 / N$ and the potential moves only one fermion to a different state [22].

In sharp contrast, in the isotropic Heisenberg model $(\Delta=1)$, due to strong antiferromagnetic fluctuations, the scattering matrix elements are drastically enhanced as shown in Fig.2. $\left|\mathcal{M}_{q}^{z}(Q=0)\right|^{2}$ scales in overall as $1 / \sqrt{N}$ and as indicated in the inset of Fig.2 in the region not close to $q=0, \pi$ the matrix element behaves approximately as

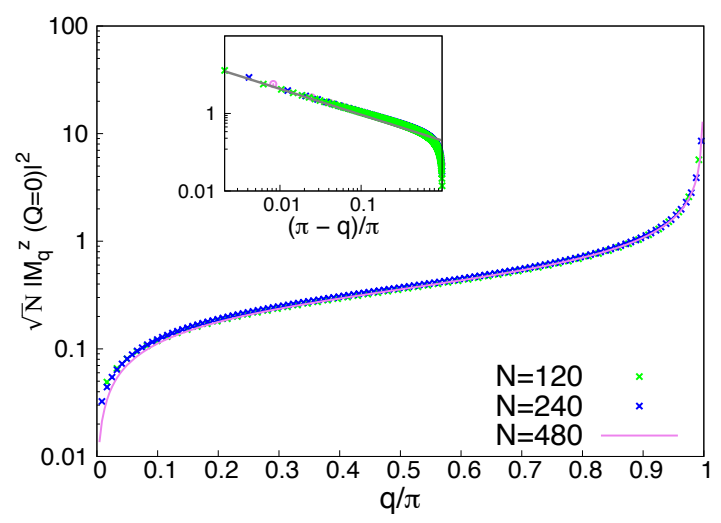

FIG. 2: Scaled $\sqrt{N}\left|\mathcal{M}_{q}^{z}(Q=0)\right|^{2}$ as a function of $q / \pi$ for $N=120,240,360,480, \Delta=1$. In the inset the asymptotic scaling of $\left|\mathcal{M}_{q}^{z}(Q=0)\right|^{2}$ with a solid line indicating the asymptote $(\pi-q)^{2 / 3}$.

$$
\left|\mathcal{M}_{q}^{z}(Q=0)\right|^{2} \sim \frac{1}{\sqrt{N}} \frac{1}{(\pi-q)^{2 / 3}} .
$$

Note that this behavior does not describe the $q=\pi$, which should not be diverging and scales differently with $N$, as will be discussed below.

The most interesting part in Fig.2 and relation (3) is that the matrix elements scale in a non-trivial fashion with $N$. In the XY model and for a $S_{n}^{z}$ potential all matrix elements scale as $1 / N$ which is the usual case in lattice scattering. On the contrary for all $\Delta \neq 0$ the matrix elements have a non trivial relation with respect to the spinon momentum and a particular scaling with respect to the number of spin sites, which is crucial to the spinon scattering.

Furthermore, using [16] and a numerical evaluation, we further address the two types of matrix elements shown in Fig.3 (and all equivalent transitions between the two spinon branches) that as we will see in the next section they play a significant role in the scattering processes. In 
the $q=\pi$ transition

$$
\left|\left\langle Q+\pi\left|S_{\pi}^{z}\right| Q\right\rangle\right|^{2} \simeq \frac{f^{z}(Q)}{N^{2 \mathcal{Z}^{2}-1}}
$$

and in the same branch flipping velocity transition

$$
\left|\left\langle\pi-Q\left|S_{\pi-2 Q}^{z}\right| Q\right\rangle\right|^{2} \simeq \frac{h^{z}(Q)}{N^{\alpha(Q)}},
$$

both corresponding to on-shell transitions. $\mathcal{Z}$ is the dressed charge introduced in $[23,24]$ and the identification has been done using the analysis in [25], since for small magnetic fields the dressed charge is $\mathcal{Z} \simeq \sqrt{\frac{\pi}{2(\pi-\gamma)}}$. In particular, $\mathcal{Z}^{2}=1$ for $\Delta=0$ and $\mathcal{Z}^{2}=1 / 2$ for $\Delta=1$. Note that this scaling of the matrix elements is also valid in the $h=0$ case, since by an analytical continuation the critical exponent $2 \mathcal{Z}^{2}$ remains the same. Furthermore, for $Q$ not close to zero $\alpha(Q) \simeq 1$ and $f^{z}(Q)$ is an almost constant function, while $h^{z}(Q)$ is a rapidly decreasing one to a constant value [19]. These types of matrix elements have been extensively studied in $[26,27]$ and the correspondence between the dressed charge and the scaling of the matrix elements has been proven analytically.

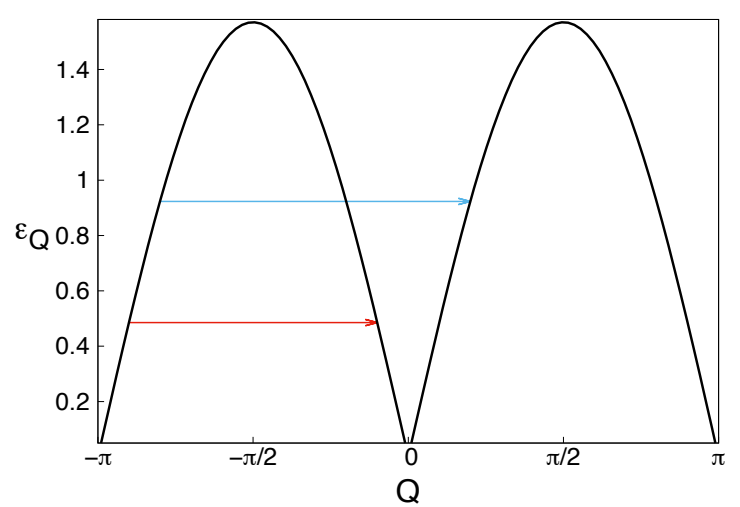

FIG. 3: Schematic description of the $Q \rightarrow \pi+Q$ transition (blue) and $Q \rightarrow \pi-Q$ same branch velocity flipping transition (red).

Next we consider the scattering of a spinon by a lattice distortion of wave-vector $q$,

$$
h_{q}=\frac{1}{\sqrt{N}} \sum_{n=1}^{N} e^{i q n} J\left(S_{n}^{x} S_{n+1}^{x}+S_{n}^{y} S_{n+1}^{y}\right)
$$

from which we can deduce the scattering from a "weak link" $V=g\left(S_{n}^{-} S_{n+1}^{+}+S_{n}^{+} S_{n+1}^{-}\right)$. Similarly to the previous case, the scaled scattering matrix element for $\Delta=1$ and the asymptotic form

$$
\left|\mathcal{M}_{q}^{p h}(Q=0)\right|^{2} \sim N^{-2 / 3} \frac{1}{(\pi-q)^{1 / 2}},
$$

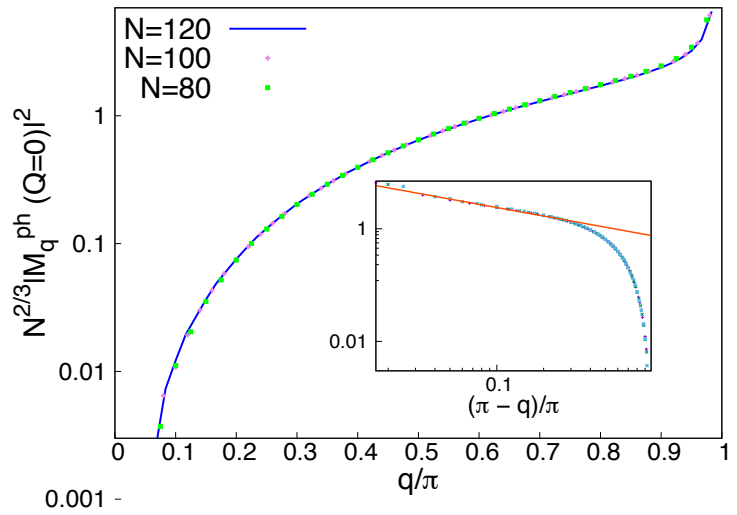

FIG. 4: Scaled $N^{\frac{2}{3}}\left|\mathcal{M}_{q}^{p h}(Q=0)\right|^{2}$ as a function of $q / \pi$. The inset shows the asymptotic scaling of $\left|\mathcal{M}_{q}^{p h}(Q=0)\right|^{2}$ as a function of $(\pi-q) / \pi$. The solid line indicates the asymptote $(\pi-q)^{1 / 2}$.

for $Q=0$ are shown in Fig.4.

Again, the dominant matrix elements for spinon scattering are a $\pi$-transition and a same branch velocity flipping matrix element,

$$
\begin{aligned}
\left|\left\langle Q+\pi\left|h_{\pi}\right| Q\right\rangle\right|^{2} & \simeq \frac{f^{p h}(Q)}{N^{a(Q)}}, \\
\left|\left\langle\pi-Q\left|h_{\pi-2 Q}\right| Q\right\rangle\right|^{2} & \simeq \frac{h^{p h}(Q)}{N} .
\end{aligned}
$$

For the isotropic $\Delta=1$ case, $a(Q)$ has a weak dependence with respect to $Q, a(Q) \simeq 0.4$ around $Q=2 \pi / 10$, while by a Jordan-Wigner transformation we can derive that for $\Delta=0$ the absolute value squared of all matrix elements scales as $1 / N$.

Finally we consider a transverse magnetic potential, $V=g S_{n}^{x}$. The main difference of this potential to the two previous ones is that it acts non-trivially only between states with $\Delta S^{z}= \pm 1$.

Similarly to the $S_{n}^{z}$ potential, as shown in Fig.5, the asymptote behaves as,

$$
\left|M_{q}^{x}(Q=0)\right|^{2} \sim \frac{1}{\sqrt{N}} \frac{1}{(\pi-q)^{2 / 3}}
$$

and the dominant matrix elements scale as,

$$
\left|\left\langle Q+\pi\left|S_{\pi}^{x}\right| Q\right\rangle\right|^{2} \simeq \frac{f^{x}(Q)}{N^{\frac{1}{2 Z^{2}}-1}} .
$$

This time, the XY model matrix elements behave nontrivially as they scale as $\sqrt{N}$ and in fact they imply a strongest scattering compared to the $0<\Delta \leq 1$ case.

Overall, the $\pi$-transitions show a strong $N$-dependence and a weak $Q$-dependence, while the 


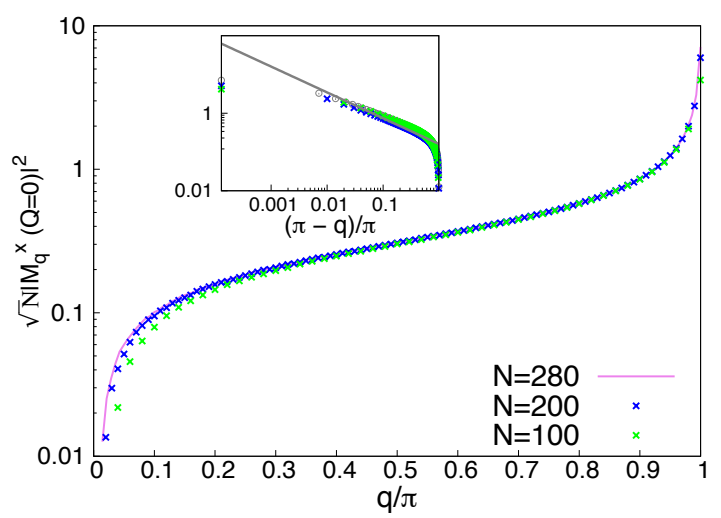

FIG. 5: Scaled $\left|M_{q}^{x}(Q=0)\right|^{2} \sqrt{N}$ vs $q$ for the isotropic model $\Delta=1$ and various $N$. The solid line in the inset shows that the asymptote scales as $(\pi-q)^{2 / 3}$.

$\pi-2 Q$ (velocity flip) transitions show a $1 / N$-dependence and a strong $Q$-dependence [19].

To close our discussion on the matrix elements, we consider an extended potential profile $V_{e x t}=\sum_{n=1}^{N} g_{n} V_{n}$, where $V_{n}$ represents one of the potentials we studied above and $g_{n}$ is the potential profile

$$
\left|\left\langle Q+q\left|V_{e x t}\right| Q\right\rangle\right|^{2}=\frac{1}{N}\left|\sum_{n=1}^{N} g_{n} e^{-i q n}\right|^{2}\left|V_{q}\right|^{2} .
$$

For example, for a segment of $m$-sites with a potential $V_{m}=\sum_{n=N / 2}^{N / 2+m-1} S_{n}^{z}$ the matrix element is given by,

$$
|<Q+q| V_{m}|Q>|^{2}=\frac{1}{N} \frac{\sin ^{2} \frac{q m}{2}}{\sin ^{2} \frac{q}{2}}\left|\mathcal{M}_{q}^{z}(Q)\right|^{2} .
$$

This form of equation can be interpreted as a "diffraction"-like pattern modified by the scattering of the spinon. For the XY model it simply becomes,

$$
|<Q+q| V_{m}|Q>|^{2}=\frac{1}{N^{2}} \frac{\sin ^{2} \frac{q m}{2}}{\sin ^{2} \frac{q}{2}} .
$$

The main message of this section is that the scattering matrix elements of the quantum many-body topological - spinon - excitations in the XXZ Heisenberg model are strongly enhanced compared to the ones in the XY model (free fermions). They show a nontrivial system size dependence and thus we expect profound differences in the scattering of spinon excitations by a potential to the generic single particle one.

\section{SCATTERING COEFFICIENTS}

We will analyze the transmission/reflection scattering coefficients of a spinon from a potential within the T- matrix approach by writing all quantities in the basis of Bethe ansatz eigenstates $|\{\lambda\}\rangle$,

$$
\begin{aligned}
T & =V \frac{1}{1-G_{0} V} \\
G_{0}(E) & =\lim _{\varepsilon \rightarrow 0} \sum_{\{\lambda\}} \frac{|\{\lambda\}\rangle\langle\{\lambda\}|}{E-E_{\{\lambda\}}+i \varepsilon} \\
V & =\sum_{\{\mu\},\{\lambda\}}\langle\{\lambda\}|V|\{\mu\}\rangle|\{\lambda\}\rangle\langle\{\mu\}| .
\end{aligned}
$$

$E_{\{\lambda\}}$ is the energy corresponding to the Bethe state $|\{\lambda\}\rangle$. Based on the discussion in the previous section for the particular scaling of the matrix elements with $N$, we write a typical matrix element in the form $\langle\{\lambda\}|V|\{\mu\}\rangle=g f_{\{\lambda\},\{\mu\}} / N^{\alpha}$ with $g$ being the potential strength and $\alpha=\alpha(\{\lambda\},\{\mu\})>0$ a scaling factor. The potential matrix $V$ belongs in a Hilbert space of dimension $\operatorname{dim} \mathcal{H}=2^{N}$ which makes the problem intractable from a computational point of view. Therefore, in order to be able to calculate the scattering coefficients for relatively long spin chains, we restrict our numerical calculations to including only the two-spinon continuum i.e. a subspace of dimension $\operatorname{dim} \mathcal{H}_{2 s p}=\frac{N}{8}(N+2)$. The calculation of the T-matrix is straightforward, we compute the matrix $1-G_{0} V$ and subsequently invert it and left multiply it by $V$. Note that for the evaluation of the Green's function $G_{0}$ we use the identity $\lim _{\varepsilon \rightarrow 0} \frac{1}{x+i \varepsilon}=P \frac{1}{x}-i \pi \delta(x)$, where $P$ stands for the Cauchy principal value part.

\section{"Free" spinon}

It is instructive to consider the scattering of a free particle on a lattice with a "spinon" dispersion relation $\varepsilon_{Q}=v_{s}|\sin Q|$ by a one-site $\delta$-like potential of strength $g$. In this case all the matrix elements are the same, $<Q^{\prime}|V| Q>=g / N$ and the transmission coefficient $\mathcal{T}_{Q, Q}$ is a function of $g / u_{Q}$ [19], $u_{Q}=d \varepsilon_{Q} / d Q$.

In Fig.6 we show that the "free" spinon transmission probability and that of a particle in a tight-binding model with dispersion relation $\varepsilon_{Q}=v_{s}(1-\cos Q)$ behave very differently. The "free" spinon transmission probability is generally a decreasing function of the energy, a property of the specific bounded spectrum. Moreover, we observe that in the linear part of the energy dispersion we have high transmission probability which is related to the fact that in a purely linear dispersion relation, i.e. a massless one dimensional Dirac equation only a phase is induced in the wavefunction and there is no reflection probability [28]. Additionally from the specific form of the spinon dispersion relation we observe that when $\varepsilon_{Q}$ decreases, $u_{Q}$ increases, which implies that $\mathcal{T}_{Q, Q}$ is an increasing function of the spinon velocity. Thus a more sensible quantity for the description of the transmission coefficient 


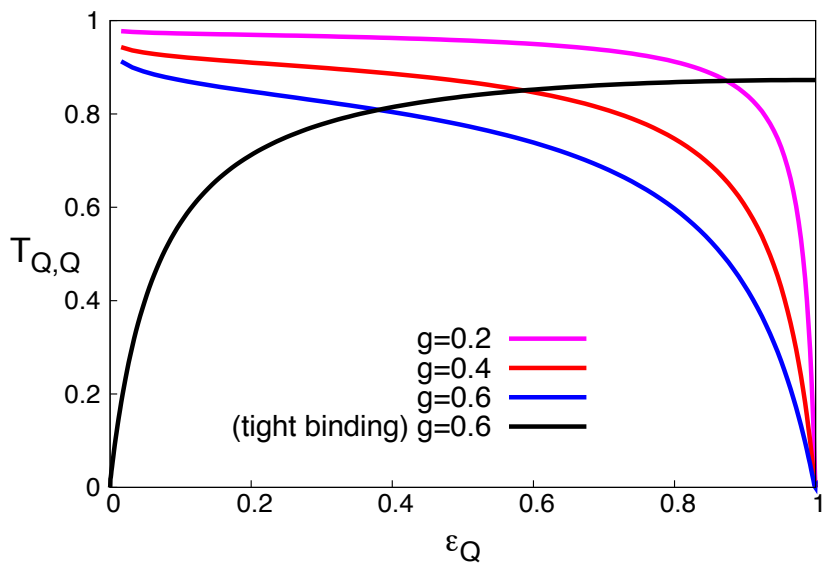

FIG. 6: $\mathcal{T}_{Q, Q}$ vs $\varepsilon_{Q}$ for a delta-like potential of strength $g$ for a "free" spinon and a particle in a tight-binding model

is the spinon velocity and not the spinon energy as in usual scattering problems.

\section{One-site longitudinal potential}

We first consider the scattering of a spinon in an oddsite chain from a one-site potential $V=g S_{n}^{z}$. In the fermionic language of the $t-V$ model [21] this would indeed correspond to the scattering of a spinless fermion from a one-site potential. In our calculation of the transmission coefficient $\mathcal{T}_{Q, Q}$ as a function of spinon energy, Fig.7, we include only the lower one spinon branch as intermediate states. For $\Delta=0$ we recover the free-spinon result of Fig.6, while for finite $\Delta$ we find a strong suppression of the transmission probability at low energies. Because of the finite size of the chain we cannot study the zero energy limit, however we expect the transmission to vanish at this limit as implied by comparing the $N=121$ and $N=301$ data at low energies. We should also note that the results are practically independent of system size, at least in this lowest branch approximation. Similar results are shown in Fig.8 for the isotropic model at different potential strengths $g$ where, as expected, the transmission is suppressed with increasing potential strength. Furthermore, as in the free spinon case, note the vanishing of the transmission at high energies, related to the zero spinon velocity at the top of the energy dispersion.

As shown in Fig.3, in an even chain there are two low energy spinon branches. In Fig.9 we find that there is a complementarity in transmission, as when $\mathcal{T}_{Q, Q}$ decreases, $\mathcal{T}_{Q, Q+\pi}$ increases. The sum of the two closely resembles the transmission of the one spinon in a odd chain. Furthermore, there is a strong size dependence of $\mathcal{T}_{Q, Q}$ which can probably best be described as exponen-

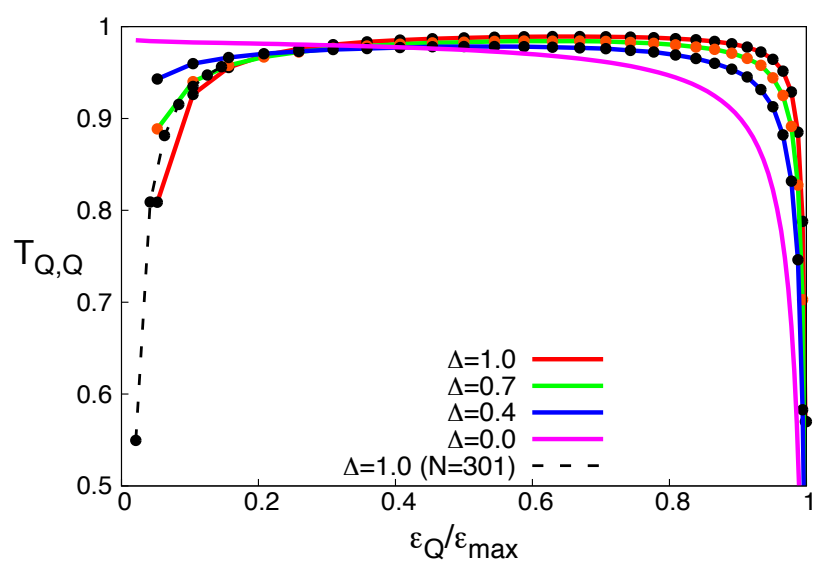

FIG. 7: $\mathcal{T}_{Q, Q}$ vs $\varepsilon_{Q}$ for various $\Delta, g=0.15$ for an odd spin chain, $N=121$. The black dashed line indicates the $N=301$ data. The solid lines are guides to the eye.

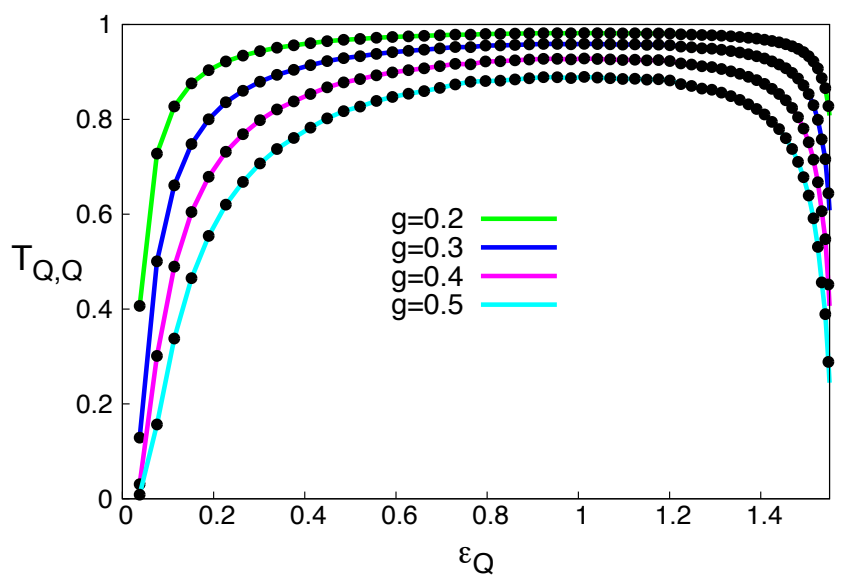

FIG. 8: $\mathcal{T}_{Q, Q}$ vs $\varepsilon_{Q}$ for an odd $N=241$ isotropic model spin chain for various $g$. The solid lines are guides to the eye.

tially decreasing with $N$. This is argued in [19] and shown in Fig.11 where for comparison a power law dependence is also plotted (not shown, there is a corresponding exponential increase of $\mathcal{T}_{Q, Q+\pi}$ ). The exponential dependence increases with $\Delta$ as shown in Fig. 10 and with $g$, Fig.11. However, the sum $\mathcal{T}_{Q, Q}+\mathcal{T}_{Q, Q+\pi}$ of transmission probabilities shows a weak size dependence and of course in the $\Delta=0$ case coincides with the one spinon in an odd chain with no size dependence. In other words, we conjecture that in the thermodynamic limit an incoming spinon from the one branch is fully transmitted/reflected in the other branch. In this calculation we have again included as intermediate states only the two lower spinon branches. As discussed below, including all the two-spinon states, only quantitatively changes this behavior. Another aspect of this transfer of transmission probability from the $\mathcal{T}_{Q, Q}$ to the $\mathcal{T}_{Q, Q+\pi}$ branch is shown in Fig.12 where we see that 


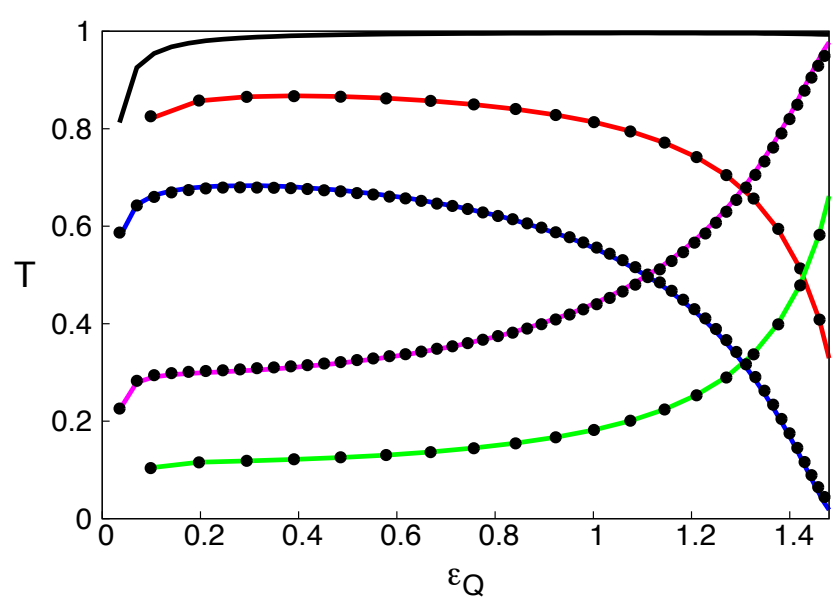

FIG. 9: $\mathcal{T}_{Q, Q}($ red $N=100$, blue $N=280)$ and $\mathcal{T}_{Q, Q+\pi}$ (green $N=100$, purple $N=280$ ) vs $\varepsilon_{Q}$ for $g=0.15$ and $\Delta=1$. The sum $\mathcal{T}_{\text {tot }}=\mathcal{T}_{Q, Q}+\mathcal{T}_{Q, Q+\pi}$ for $N=280$ is indicated by a solid black line. The solid lines represent the analytical results while the dots the numerical data.

$\mathcal{T}_{Q, Q+\pi}$ increases with potential strength.

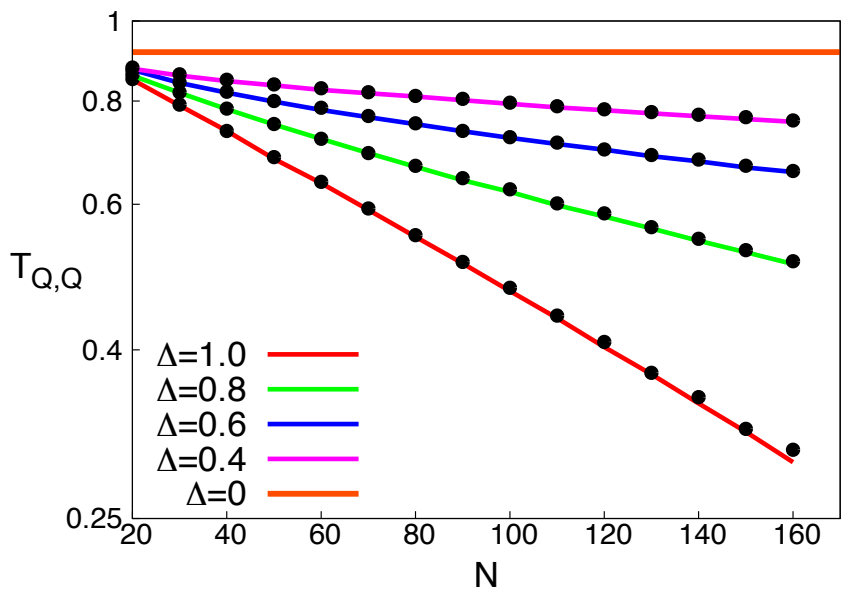

FIG. 10: $\log \mathcal{T}_{\mathcal{Q}, \mathcal{Q}}$ vs $N$ for $V=g S_{n}^{z}, g=0.2$ for constant energy $\varepsilon_{Q} / v_{s}=\sin (2 \pi / 10)$ and

$\Delta=0.4,0.6,0.8,1.0$ The solid lines represent the analytical approximation [19], while the dots represent the numerical data. The horizontal represents the

$$
\Delta=0 \text { case. }
$$

Based on the integrable structure of the Heisenberg model we can understand these results from first principles [19]. Re-summing to all orders the most important on-shell matrix elements, $|Q\rangle \rightarrow|Q+\pi\rangle,|\pi-Q\rangle$ described in the previous section, we obtain a fairly good description of the transmission probabilities (even quantitative in the weak coupling limit). It is easily proved that these transitions result in a monotonically decreasing (increasing) transmission probability $\mathcal{T}_{Q, Q}\left(\mathcal{T}_{Q, \pi+Q}\right)$
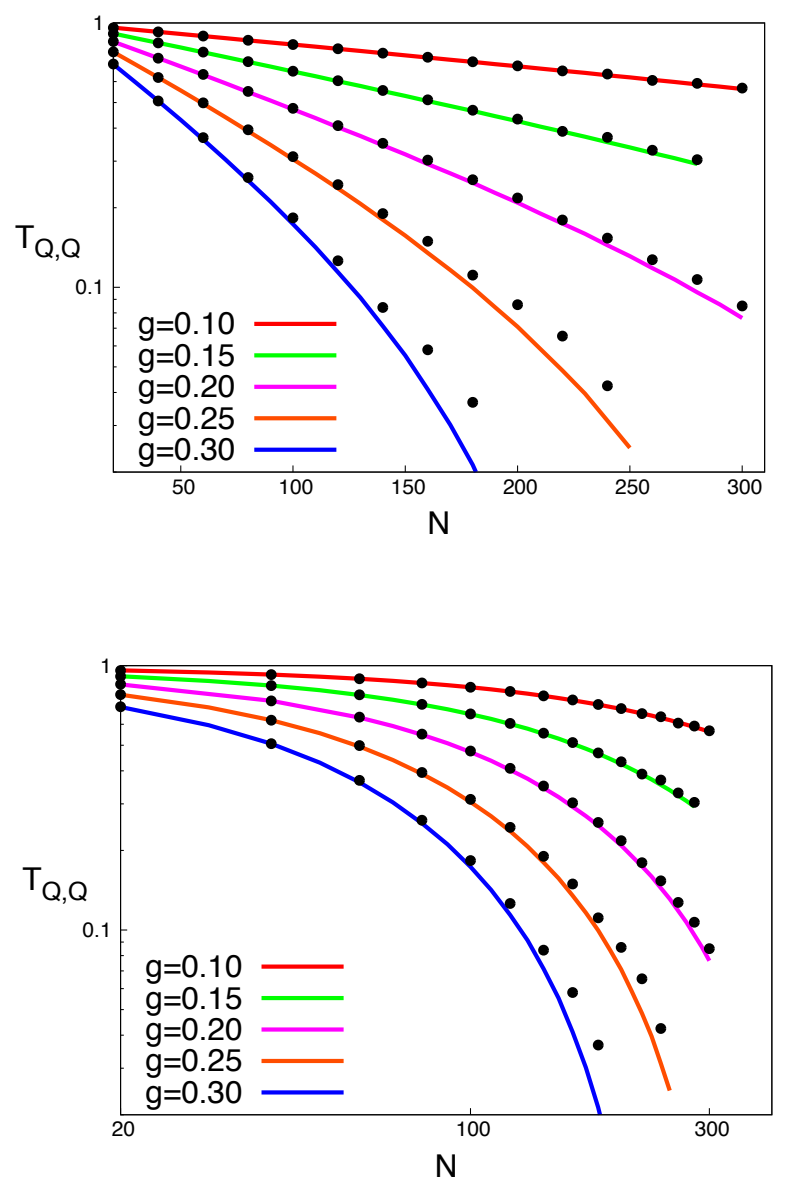

FIG. 11: $\log \mathcal{T}_{Q, Q}$ vs $N$ (top) and $\log \mathcal{T}_{Q, Q}$ vs $\log N$ (bottom) for $V=g S_{n}^{z}$ for the isotropic model $\Delta=1$ and energy $\varepsilon_{Q}=\frac{\pi}{2} \sin (2 \pi / 10) \simeq 0.92$. The solid lines represent the analytical approximation [19], while the dots represent the numerical data.

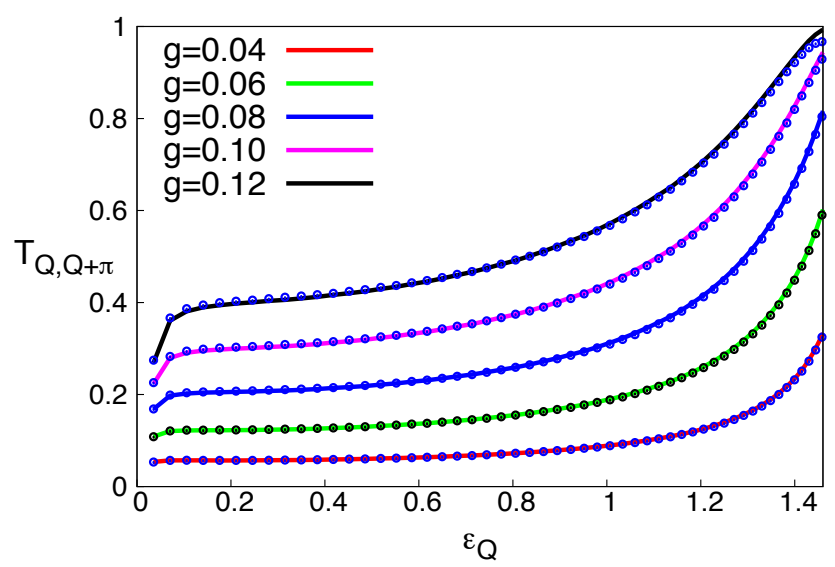

FIG. 12: $\mathcal{T}_{Q, Q+\pi}$ vs $\varepsilon_{Q}$ as a function of $g$ for $N=280$. The solid lines are guides to the eye. 
with spin chain length $N$. We expect this behavior to be generic in one dimensional spin chains, simply here, the integrability of the model allows us to explicitly evaluate the corresponding exponents.

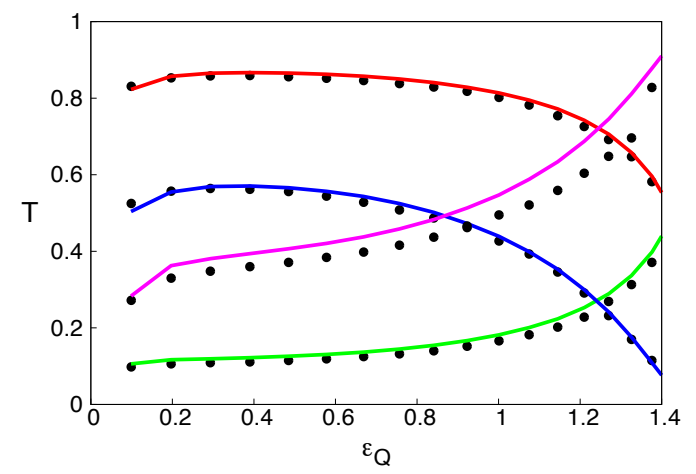

FIG. 13: $\mathcal{T}_{Q, Q}($ red $g=0.1$, blue $g=0.2)$ and $\mathcal{T}_{Q, Q+\pi}$ (green $g=0.1$, purple $g=0.2$ ) vs $\varepsilon_{Q}$ for $N=100$ and $\Delta=1$. The solid lines are produced by including only the lower branch while the dots represent the numerical data obtained by including the whole two-spinon continuum.

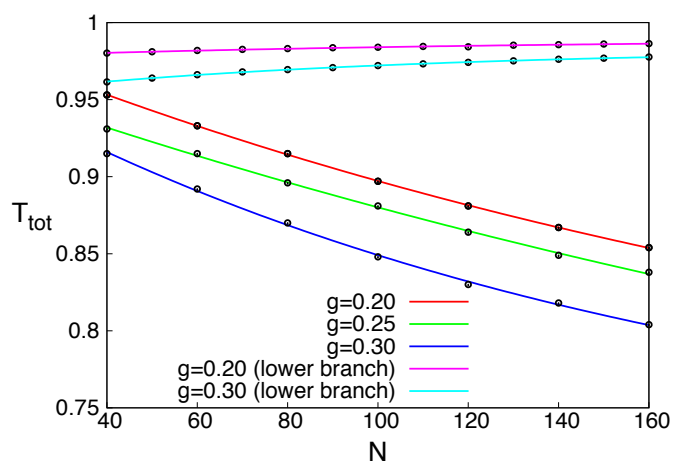

FIG. 14: $\mathcal{T}_{\text {tot }}$ versus $N$ for the $V=g S_{n}^{z}$ potential $\Delta=1$ and $\varepsilon_{Q} \simeq 0.923$ including the two-spinon continuum along the lower branch data. The dots represent the numerical data, while the solid lines represent the fitted curve $\mathcal{T}_{\text {tot }}=A \exp \left(-B f^{z}(Q) g^{2} N / u_{Q}^{2}\right)+C$.

By a numerical fit in Figs.10,11 we find that a useful quantity for the description of the scattering process is $g_{\text {eff }}=g N^{1-\mathcal{Z}^{2}}$ and that for $\varepsilon_{Q}$ not close to zero the transmission coefficient behaves as

$$
\mathcal{T}_{Q, Q} \simeq e^{-a\left(g_{\text {eff }} / u_{Q}\right)^{2}}
$$

which holds for $g_{e f f} / u_{Q}<<1$. Thus for the isotropic Heisenberg model $(\gamma=0, \Delta=1)$ which is the most experimentally relevant $\mathcal{T}_{Q, Q} \simeq e^{-a\left(g / u_{Q}\right)^{2} N}$. Although this approach does not offer an analytical solution of the scattering problem, using the framework of integrability we derived a connection between the transmission coefficients and $\theta_{z z}=2 \mathcal{Z}^{2}$, the critical exponent of the ground state's correlation function $\left\langle 0\left|s_{1}^{z} s_{n+1}^{z}\right| 0\right\rangle$ dominant oscillatory part. Predicted by CFT and Bethe Ansatz calculations $[16,23]$, it offers a qualitative description of the scattering process.

Note that this approximation gives reasonable results even though we have performed a rough elimination of most of the intermediate matrix elements. On the other hand from the specific form of the transmission probability of the "free" spinon model we observe that the dominant behavior is given by the on-shell matrix elements and the rest of the matrix $V$ serves as a correction, which justifies the reasoning for the above approximation. Of course, as we see in Fig.11, it is a weak coupling approximation, albeit a very good one, that becomes increasingly unreliable in the strong $g$ coupling limit. Even more, in the strong coupling $g / u_{Q}>>1$ limit (e.g. $Q \rightarrow \pm \pi / 2$ ) the numerical T-matrix approach we are using often does not converge at all.

Finally, to improve on the lower branch approximation we include all the two-spinon Cloiseaux-Pearson [18] states which forces us however to study rather small size spin chains as the space of intermediate states increases as $N^{2}$. As shown in Figs. 13 and 14 it results to only quantitative differences with most of the effect coming from the $\mathcal{T}_{Q, Q+\pi}$ transition. We estimate the $N \rightarrow+\infty$ extrapolated value of $\mathcal{T}_{\text {tot }}$ to be reduced by about 30 percent from the value of the lower branch data. We should note however that the two-spinon continuum data become increasingly sensitive with system size to details of the calculation e.g. separation of the real and imaginary part in the T-matrix numerical evaluation.

Comparing the even and odd site case, we found an interesting topological effect. In the odd chains, in our one-spinon study where the spectrum is two-fold degenerate, we find a rather regular behavior of scattering coefficients. In the even chains, due to the topological twospinon constraint, we have a four-fold degenerate spectrum that, together with the singular $\pi$-transition, implies a transfer of transmission probability between the two spinon branches. Thus, in the spinon scattering, we have an interplay of the topological character and the singular matrix elements of a critical system.

\section{Spin-phonon potential}

The spin-phonon interaction is described by a one-link potential of the form

$$
V=g\left(S_{n}^{-} S_{n+1}^{+}+S_{n}^{+} S_{n+1}^{-}\right) .
$$

In Fig.15 the numerical calculation for an even-site chain shows that $\mathcal{T}_{Q, Q} \rightarrow 0$ as $N$ increases. Similarly to the previous case, we obtain an approximate analytical result by using the dominant matrix elements that 
were described in the previous section. In particular, the monotonicity of the scaling factors implies that the transmission and reflection coefficients will be scale invariant for $\Delta=0$ while on the contrary, for $0<\Delta \leq 1 \mathcal{T}_{Q, Q} \rightarrow 0$ as $N$ increases. Moreover, the relation of the scattering coefficients to the spinon energy $\varepsilon_{Q}$ is very similar to that of a longitudinal magnetic potential as was depicted in Fig.9.

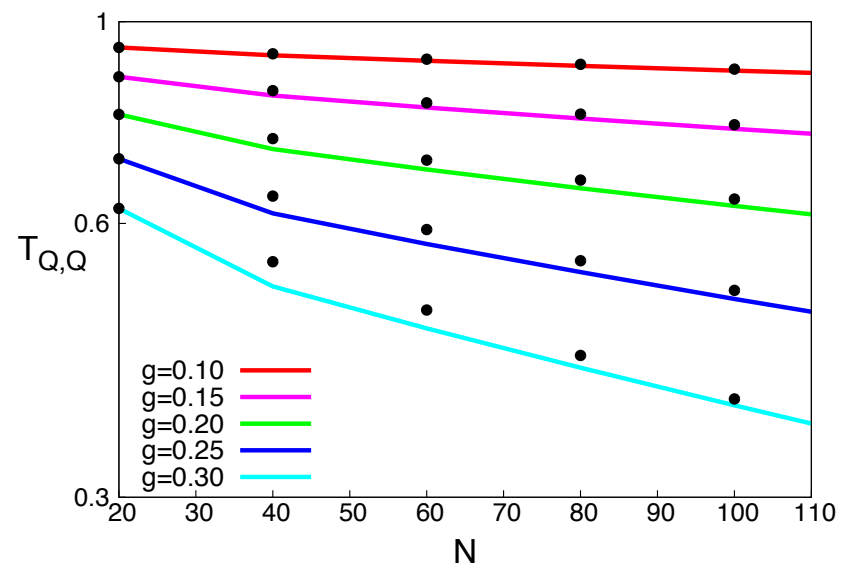

FIG. 15: $\log \mathcal{T}_{Q, Q}$ vs $N$ for a one-link spin-phonon potential $V, \varepsilon_{Q} \simeq 0.92$ and $g=0.1,0.15,0.2,0.25,0.3$. Solid lines prediction based on the dominant matrix elements [19].

\section{Transverse potential}

We now turn to a transverse magnetic potential, $V=$ $g S_{n}^{x}$. The main difference of this potential to the two previous ones we studied is that it acts non trivially only between states with $\Delta S^{z}= \pm 1$. We will restrict ourselves to transitions between the $S^{z}=1$ and $S^{z}=2$ magnetization sectors.

Fig.16 shows that similarly to the previous cases, for an even-site chain $\mathcal{T}_{Q, Q} \rightarrow 0$ as $N$ increases. Again the dependence is probably best be described as exponential, as argued in [19] and by comparison with a power law one. However, this time we find that this holds also for $\Delta=0$ and in fact the scattering increases as $\Delta$ decreases, which is the opposite to what happened in the previous cases. Again we can obtain a qualitative explanation of this behavior by using the fact $[26,27]$ that the dominant matrix element approximately scales as $\theta_{-+}=\frac{1}{2 \mathcal{Z}^{2}} \simeq \frac{\pi-\gamma}{\pi}$ which is the dominant critical exponent of the ground state correlation $\left\langle 0\left|\sigma_{1}^{-} \sigma_{n+1}^{+}\right| 0\right\rangle$. By re-summation [19] and the monotonicity of the critical exponents with respect to $\Delta$ one can argue that $\mathcal{T}_{Q, Q} \rightarrow 0$ for $0<\Delta \leq 1$. Nevertheless, a full scale analysis of the matrix elements should be done in order to give a definite answer. Similarly to the previous cases, by defining $g_{\text {eff }} \equiv g N^{1-\frac{1}{4 \mathcal{Z}^{2}}}$
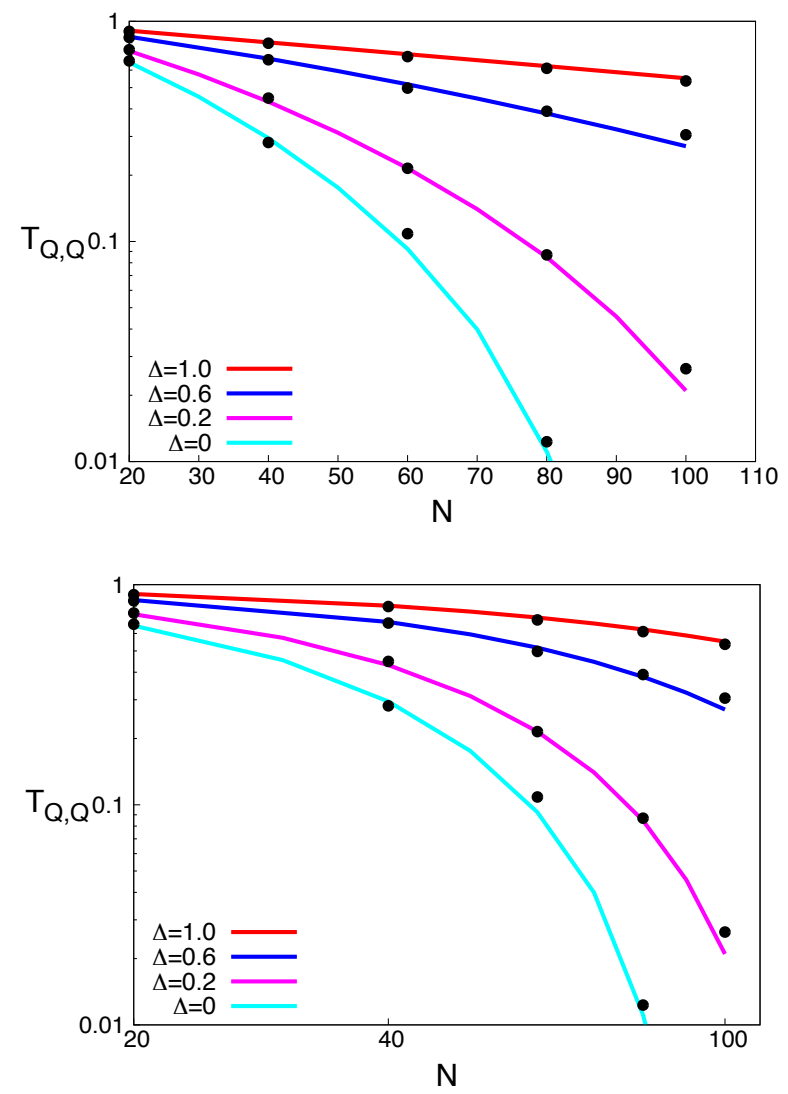

FIG. 16: $\log \mathcal{T}_{Q, Q}$ vs system size $N$ (top) and $\log \mathcal{T}_{Q, Q}$ vs system size $\log N$ for a one-site transverse potential $V=g S_{n}^{x}, g=0.2, \varepsilon_{Q}=v_{s} \sin \left(\frac{2 \pi}{10}\right)$ and

$\Delta=1,0.6,0.2,0.0$. Solid lines are the prediction considering the dominant matrix elements [19].

implying $g_{e f f}=g \sqrt{N}$ for $\Delta=1$ (isotropic model) and $g_{\text {eff }}=g N^{3 / 4}$ for $\Delta=0$ (XY model), we conclude that

$$
\mathcal{T}_{Q, Q} \simeq e^{-f^{x}(Q)\left(g_{e f f} / u_{Q}\right)^{2}}
$$

in the region $g_{\text {eff }} /\left(4 u_{Q}^{2} / f^{x}(Q)\right)<<1$, a behavior which agrees well with the numerical data.

\section{Extended potential}

Finally, we consider the spinon scattering from an extended potential

$$
V_{e x t}=\sum_{n=1}^{m} g_{n} V_{n}
$$

By the numerical procedure presented earlier, we can calculate the transmission probability for an arbitrary potential profile $\left\{g_{n}\right\}$ in the two-spinon continuum approximation. We start with the scattering of a spinon in 
an odd chain by a two-site longitudinal potential, a case analogous to Fig.7 for a on-site potential. In Fig.17 we see a remarkable difference at low energies where there is complete transmission. This situation is consistent with the well known "cutting" and "healing" [29, 30] effect in one dimensional correlated systems and spin chains, where one weak-link is cutting a chain at low energies while two weak-links are healed. This effect leads to a finite conductance with a power law dependence on the temperature due to thermal effects.

Here, we can understand the results of extended potentials by considering the "diffraction" relation (12). For an $m=2$ longitudinal potential in an odd chain, at low energies $Q \rightarrow 0$, the $q=\pi-2 Q$ scattering matrix element vanishes leading to total transmission. Following the same argument, we also find that for an even chain with an $m=2$ longitudinal potential the transfer of transmission probability from $\mathcal{T}_{Q, Q}$ to $\mathcal{T}_{Q, Q+\pi}$ found in Fig.7 is now totally suppressed as the $q=\pi$ matrix element vanishes. Following the same line of re-summation of dominant matrix elements and by taking into account the corresponding "diffraction" factor allows us to understand the transmission by extended potentials.

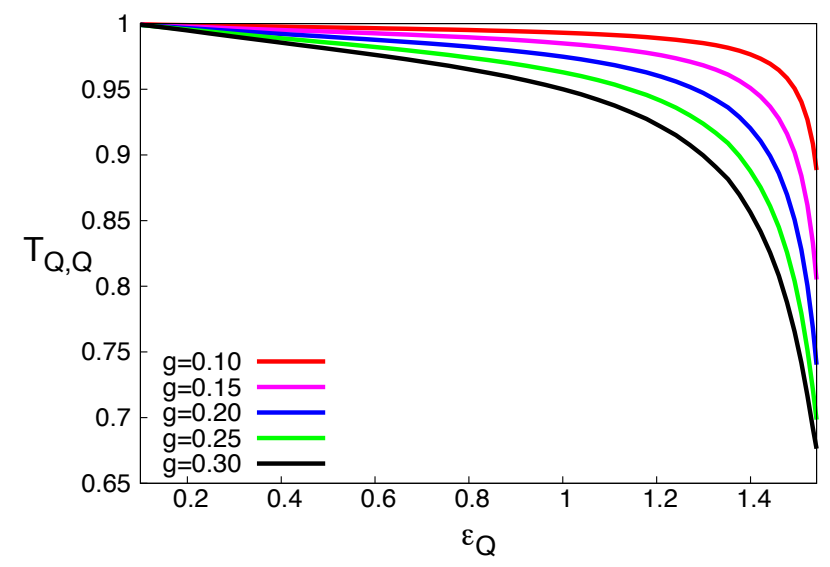

FIG. 17: $\mathcal{T}_{Q, Q}$ vs spinon energy $\varepsilon_{Q}$ for a potential $V=g\left(S_{n}^{z}+S_{n+1}^{z}\right), N=201$.

\section{CONCLUSIONS}

Using the Bethe ansatz method and the T-matrix approach, we have studied the scattering of a spinon from prototype potentials. Three main features emerged from this study; first, we are considering a quantum manybody problem, so in principle outgoing states with creation of spinons or "electron-hole" pairs is possible although we expect from the scattering matrix elements that these processes have lower probability. We have limited our study to outgoing states with the same number of spinons as the incoming state. Second, we can qualitatively account for the transmission probabilities by re-summing the dominant scattering elements. Their dependence on the size of the spin chain is given by the critical exponents characterizing the anisotropic Heisenberg model. Thus, we linked the scattering to the critical properties of this integrable model and we evaluated them by the Bethe ansatz method. It is an open, technically very difficult, question whether including all intermediate states $O\left(2^{N}\right)$ would qualitatively change the present picture. Third, we have found an intriguing topological effect as, in an even chain there is complete transfer of the incoming spinon transmission probability from the one branch of the dispersion to the other branch. At the moment, in a macroscopic open chain the role of this odd-even effect is ambiguous. Further study is necessary to clarify it, presumably including further outgoing states, e.g. three-spinon states in odd chains. Note that, several experimental and theoretical studies [32] have addressed the physical effect of even vs. odd chain length in the thermodynamic properties of finite size chains.

Along the line of dominant matrix elements, we analyzed a basic difference in the scattering coefficients of longitudinal and weak link potentials to those of a transverse potential. We also discussed extended potentials where, a drastic dependence of scattering coefficients on the potential extent, we attributed it to a geometric "diffraction" factor and dominant scattering matrix elements. These results are consistent with previous studies on "cutting-healing" in 1D correlated systems [29-31].

Considering experiment, we studied the problem of a spinon excited above the ground state and scattering from a potential. Although we have not addressed any particular experiment, our study would provide key elements in the interpretation of far-out of equilibrium experiments as well as thermal transport ones. For instance zero temperature tunneling studied by a "Landauer" type approach or spinon transport probed e.g. by terahertz $2 \mathrm{D}$ coherent spectroscopy [33] experiments.

\section{ACKNOWLEDGMENTS}

This work was supported by the European Union Program No. FP7-REGPOT-2012-2013-1 under Grant No. 316165, the Deutsche Forschungsgemeinschaft through Grant HE3439/13, the Alexander von Humboldt Foundation, the Hellenic Foundation for Research and Innovation (HFRI) and the General Secretariat for Research and Technology (GSRT), under the HFRI PhD Fellowship grant KA4819. A.P. acknowledges valuable discussions with P. Lambropoulos, N. Kitanine and J.S. Caux. X.Z. acknowledges fruitful discussions with H. Tsunetsugu, A. Klümper, T. Tomaras, C. Hess, B. Büchner, A. Chernyshev, S. White and the hospitality of the Institute for Solid State Physics - U. Tokyo and University of Irvine. 
[1] C. Hess, Eur. Ph. J. Special Topics 151, 73 (2007); arXiv:cond-mat/1805.01746.

[2] X. Zotos, F. Naef, and P. Prelovšek, Phys. Rev. B55, 11029 (1997).

[3] A. Klümper and K. Sakai, J. Phys. A35, 2173 (2002).

[4] E. Shimshoni, N. Andrei and A. Rosch, Phys. Rev. B68, 104401 (2003).

[5] K. Louis, P. Prelov̌sek and X. Zotos, Phys. Rev. B74, 235118 (2006).

[6] A.L. Chernyshev, A.V. Rozhkov, Phys. Rev. Lett. 116, 017204 (2016).

[7] X. Zotos, J. Stat. Mech. 103101 (2017).

[8] G.E.W Bauer, E. Saitoh and B. J. van Wees, Nature Mat. 11, 391 (2012).

[9] L.J. Cornelissen, J. Liu, R.A. Duine, J. Ben Youssef and B.J. van Wees, Nature Phys. 11, 1022 (2015); and references therein in this rapidly expanding field.

[10] D. Hirobe, M. Sato, T. Kawamata, Y. Shiomi, K. Uchida, R. Iguchi, Y. Koike, S. Maekawa and E. Saitoh, Nature Phys. 13, 30 (2017); J. Appl. Phys., 123, 123903 (2018).

[11] M. Takahashi 2005 Thermodynamics of One-dimensional Solvable Models (Cambridge University Press).

[12] K. Louis, and C. Gros, Phys. Rev. B67, 224410 (2003).

[13] K. Sakai, and A. Klümper, J. Phys. Soc. Jpn. Suppl. 74, 196 (2005).

[14] C. Psaroudaki and X. Zotos 2016 J. Stat. Mech. 063103.

[15] L.d. Faddeev and L.A. Takhtajan, Phys. Lett. 85A, 375 (1981)

[16] N. Kitanine, J.M. Maillet, V. Terras, Nucl. Phys. B554, 647 (1999).

[17] J.S. Caux, R. Hagemans and J. M. Maillet, J. Stat. Mech., P09003 (2005).

[18] J. des Cloizeaux and J.J. Pearson, Phys. Rev. 128, 2131 (1962).
[19] See Supplemental Material for further details on the dominant matrix elements and scattering theory calculations.

[20] For our convention for $S_{q}^{z},|<Q+q| S_{q}^{z}|Q>|^{2}=N \mid<$ $Q+q\left|S_{n}^{z}\right| Q>\left.\right|^{2}$

[21] See, e.g., V. Emery, in Highly Conducting OneDimensional Solids, edited by J.T. Devreese et al. Plenum Press, New York, 1979, p. 247.

[22] D. Biegel, M. Karbach, Michael, G. Müller, and K. Wiele, Phys Rev B69, 174404 (2004).

[23] V.E. Korepin, Teor. Mat. Fiz. 41, 169 (1979).

[24] N.M. Bogolyubov, A.G. Izergin and V.E. Korepin, Nucl.Phys. B275, 687 (1986).

[25] M. Dugave, F. Göhmann and K. K. Kozlowski SIGMA 10, 043 (2014).

[26] N. Kitanine, K. K. Kozlowski, J. M. Maillet, N. A. Slavnov and V. Terras, J. Stat Mech. P05028 (2011).

[27] N. Kitanine, K. K. Kozlowski, J. M. Maillet, N. A. Slavnov and V. Terras, J. Stat. Mech. P05011 (2014).

[28] T. R. Robinson, American Journal of Physics 80, 141 (2012).

[29] C.L. Kane, M.P.A. Fisher, Phys. Rev. Lett.68, 1220 (1992); Phys. Rev. B46, 15233 (1992).

[30] S. Eggert, I. Affleck, Phys. Rev. B46, 10866 (1992).

[31] A. Metavitsiadis, X. Zotos, O.S. Barišić, P. Prelovšek, Phys. Rev. B81, 205101 (2010).

[32] J. Sirker, S. Fujimoto, N. Laflorencie, S. Eggert and I. Affleck, J. Stat. Mech. P02015 (2008) and references therein.

[33] Y. Wan and N.P. Armitage, arXiv:1905.11420.

[34] H. Bethe, Z. Phys. 71, 205 (1931).

[35] V. E. Korepin, N. M. Bogoliubov and A. G. Izergin, Quantum Inverse Scattering Method and Correlation Functions, Cambridge University Press.

[36] Caux, J.-S., R. Hagemans, and J. M. Maillet, J. Stat. Mech. P09003 (2005).

[37] E. Merzbacher, "Quantum Mechanics" (John Wiley \& Sons, 1998).

\section{SUPPLEMENTARY MATERIAL: SCATTERING OF SPINON EXCITATIONS BY POTENTIALS IN THE 1D HEISENBERG MODEL}

\section{Dominant Matrix elements}

In this section we briefly describe the prescription for the calculation of the matrix elements of the anisotropic Heisenberg spin chain, the dominant matrix elements in the scattering processes and their relation to the critical exponents that appear in the correlation functions.

We begin by using the fact that in the XXZ spin chain, the total magnetization commutes with the Hamiltonian, which lead us to partition the Hilbert space into subspaces of fixed magnetization, determined from the number of reversed spins $M$. For simplicity we take an even number of spin sites and $2 M \leq N$. Therefore all the eigenstates in each subspace are fully described by a set of rapidities $\left\{\lambda_{k}\right\}_{k=1}^{M}$, which correspond to solutions of the Bethe equations $[34,35]$.

$$
\arctan \left[\frac{\tanh \left(\lambda_{k}\right)}{\tan (\gamma / 2)}\right]-\frac{1}{N} \sum_{j=1}^{M} \arctan \left[\frac{\tanh \left(\lambda_{j}-\lambda_{k}\right)}{\tan (\gamma)}\right]=\frac{\pi}{N} I_{k},
$$

where $\gamma=\arccos \Delta . \quad\left\{I_{k}\right\}_{k=1}^{M}$ are different integers or half-integers, defined modulo $N$, which are the analog of quantum numbers due to the fact that each particular choice of a set $\left\{I_{j}\right\}_{k=1}^{M}$ uniquely specifies a Bethe eigenstate. Note that the ground state is given by the configuration $\left\{-\frac{M+1}{2}+k\right\}_{k=1}^{M}$. 
The energy and momentum of a state parametrized by the set of rapidities $\left\{\lambda_{k}\right\}_{k=1}^{M}$ or equivalently the set of quantum numbers $\left\{I_{k}\right\}_{k=1}^{M}$ are given by,

$$
\begin{gathered}
E_{\{\lambda\}}=J \sum_{k=1}^{M} \frac{-\sin ^{2} \gamma}{\cosh 2 \lambda_{k}-\cos \gamma}-h\left(\frac{N}{2}-M\right) \\
Q_{\{\lambda\}}=\sum_{k=1}^{M} i \ln \frac{\sinh \left(\lambda_{k}+i \gamma / 2\right)}{\sinh \left(\lambda_{k}-i \gamma / 2\right)}=\pi M+\frac{2 \pi}{N} \sum_{k=1}^{M} I_{k} .
\end{gathered}
$$

From now on, we redefine $E_{\{\lambda\}}, Q_{\{\lambda\}}$ to be above the lowest spinon energy and momentum respectively and those belonging to the lowest branch of the two spinon continuum we write them as $\varepsilon_{Q}$ and $Q$ respectively. For $0<\Delta \leq 1$ the two spinon spectrum is defined as the set of all the real solutions, with dimension $N(N+2) / 8$.

Kitanine et al. [16, 36], via an algebraic procedure, calculated the form factors for the anisotropic spin chain and successfully reduced complicated matrix elements between Bethe states for local spin operators to determinant expressions. The matrix elements for the longitudinal, transverse and spin-phonon potential are given by the expression $\left\langle\{\lambda\}\left|V_{n}\right|\{\mu\}\right\rangle=\frac{F_{n}^{a}(\{\lambda\},\{\mu\})}{\sqrt{\mathcal{N}(\{\lambda\}) \mathcal{N}(\{\mu\})}}$, where $a=z, x, p h, F_{n}^{a}(\{\lambda\},\{\mu\})$ is the form factor and $\mathcal{N}(\{\lambda\})=\langle\{\lambda\} \mid\{\lambda\}\rangle$ the norm of the unnormalized Bethe state $|\{\lambda\}\rangle$ as fully described in [16]. Using these expressions we calculate all the matrix elements of the potential matrix written in the Bethe Ansatz basis,

$$
V=\sum_{\{\lambda\},\{\mu\}}\langle\{\lambda\}|V|\{\mu\}\rangle|\{\lambda\}\rangle\langle\{\mu\}|,
$$

corresponding to longitudinal, transverse and spin-phonon potentials.

$$
\text { Longitudinal potential } V=g S_{n}^{z}
$$

The dominant matrix elements for the longitudinal magnetic potential are characterized by a $\pi$-transfer process $\left|\left\langle Q+\pi\left|S_{\pi}^{z}\right| Q\right\rangle\right|^{2}$ and by a velocity-flipping $\left|\left\langle\pi-Q\left|S_{\pi-2 Q}^{z}\right| Q\right\rangle\right|^{2}$ process in the same branch of the one-spinon spectrum. We have numerically evaluated the $\pi$-transfer and the same branch velocity-flipping matrix elements as depicted in Figs.18 and 19, scaling as,

$$
\begin{aligned}
\left|\left\langle Q+\pi\left|S_{\pi}^{z}\right| Q\right\rangle\right|^{2} & \simeq \frac{f^{z}(Q)}{N^{2 \mathcal{Z}^{2}-1}} \\
\left|\left\langle\pi-Q\left|S_{\pi-2 Q}^{z}\right| Q\right\rangle\right|^{2} & \simeq \frac{h^{z}(Q)}{N},
\end{aligned}
$$

and determined $f^{z}(Q), h^{z}(Q)$. Numerically, $f^{z}(Q)$ is an almost constant function with respect to the spinon momentum $Q$ (inset Fig. 21), while $h^{z}(Q)$ is a rapidly decreasing one. Note that the scaling of this $\pi$ transfer matrix element has been analytically studied by Kitanine et al. in [26, 27].

Transverse potential $V=g S_{n}^{x}$

The dominant matrix elements for the transverse magnetic potential are characterized by a $\pi$-transfer $\left|\left\langle Q+\pi\left|S_{\pi}^{x}\right| Q\right\rangle\right|^{2}$ which we numerically evaluate as depicted in Fig.20 and scales as [26, 27],

$$
\left|\left\langle Q+\pi\left|S_{\pi}^{x}\right| Q\right\rangle\right|^{2} \simeq \frac{f^{x}(Q)}{N^{\frac{1}{2 \mathcal{Z}^{2}}-1}}
$$

Note for values of $\Delta$ close to one, $f^{x}(Q)$ can be considered constant (inset Fig. 21), while for values of $\Delta$ close to zero it is a monotonically decreasing function. 


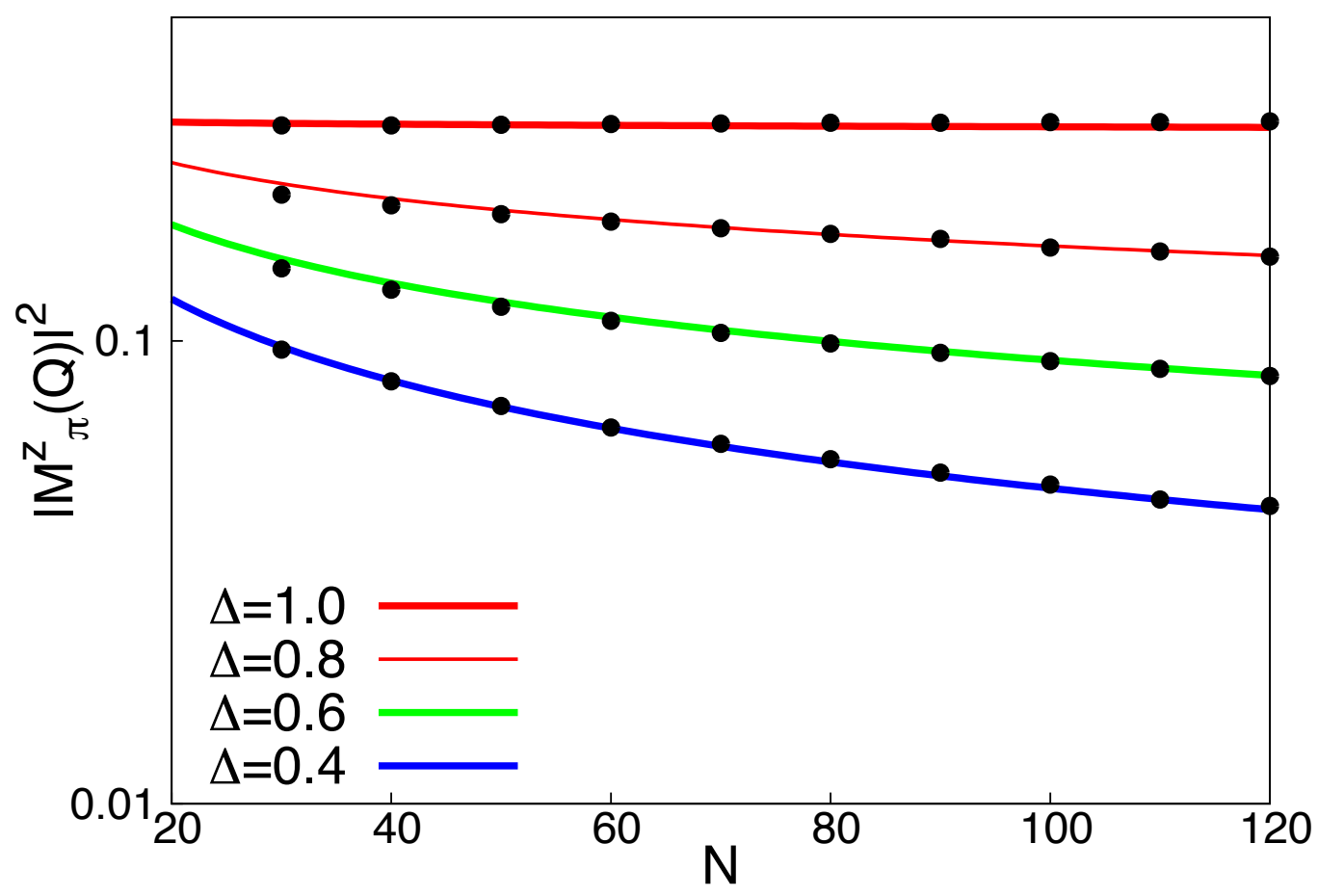

FIG. 18: $\left|\left\langle Q+\pi\left|S_{\pi}^{z}\right| Q\right\rangle\right|^{2}$ versus $N$ for $\Delta=1,0.8,0.6,0.4$, for $Q=2 \pi / 10$. The solid lines correspond to the fitted curve of the form $\left|\left\langle Q+\pi\left|S_{\pi}^{z}\right| Q\right\rangle\right|^{2} \simeq 1 / N^{2 \mathcal{Z}^{2}-1}$ while the dots are the numerical data

\section{Spin-phonon interaction}

Finally we discuss a spin-phonon interaction. Similarly with the longitudinal magnetic potential, the dominant matrix elements are characterized by a $\pi$-transfer $\left|\left\langle Q+\pi\left|V_{\pi}\right| Q\right\rangle\right|^{2}$ and by a velocity-flipping $\left|\left\langle\pi-Q\left|V_{\pi-2 Q}\right| Q\right\rangle\right|^{2}$ in the same branch of the one-spinon spectrum.

Similarly, we obtain that the $\pi$-transfer and the same branch velocity flipping matrix elements behave as,

$$
\begin{aligned}
\left|\left\langle Q+\pi\left|V_{\pi}\right| Q\right\rangle\right|^{2} & \simeq \frac{f^{p h}(Q)}{N^{\alpha}} \\
\left|\left\langle\pi-Q\left|V_{\pi-2 Q}\right| Q\right\rangle\right|^{2} & \simeq \frac{h^{p h}(Q)}{N},
\end{aligned}
$$

with $\alpha \simeq 0.4$ for $Q=2 \pi / 10$ and small corrections with respect to $Q$. Note that similarly to the longitudinal potential, $h^{p h}(Q)$ is a rapidly decreasing function for $\Delta>0$ while for $\Delta=0$ is constant and equal to one. Moreover, $f^{p h}(Q)$ and $h^{p h}(Q)$ are symmetric with respect to $\pi / 2$.

\section{Scattering Theory}

In this section we give a short description of the T-matrix approach for evaluating the transmission and reflection probability of spinon scattering from magnetic and non-magnetic potentials. The Lippmann-Schwinger equation is

$$
|\psi\rangle=\left|\psi_{0}\right\rangle+\left(E-H_{0}\right)^{-1} V|\psi\rangle,
$$

where $\left|\psi_{0}\right\rangle$ is the unperturbed incident eigenstate of the $H_{0}$ Hamiltonian and $V$ is the scattering potential. By iteratively solving the Lippmann-Schwinger equation we formally obtain the Born Series

$$
|\psi\rangle=\sum_{k=0}^{\infty}\left(G_{0} V\right)^{k}\left|\psi_{0}\right\rangle .
$$




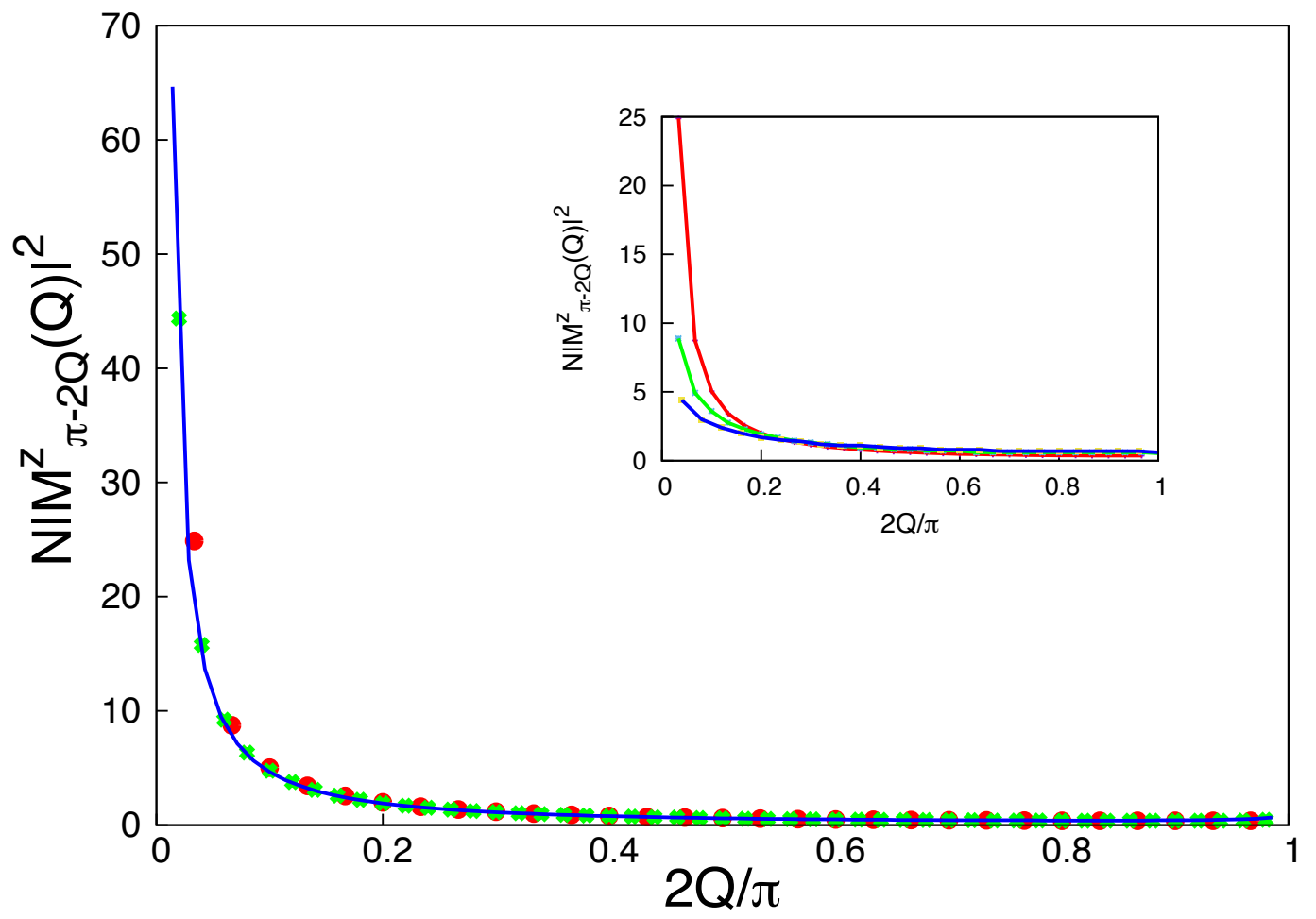

FIG. 19: Scaled $N\left|\left\langle\pi-Q\left|S_{\pi-2 Q}^{z}\right| Q\right\rangle\right|^{2}$ versus $Q$ for $\Delta=1$ and various $N$. The matrix elements are symmetric with respect to $Q=\pi / 2$. The inset shows $N\left|\left\langle\pi-Q\left|S_{\pi-2 Q}^{z}\right| Q\right\rangle\right|^{2}$ versus $Q$ for $\Delta=1,0.6,0.4$.

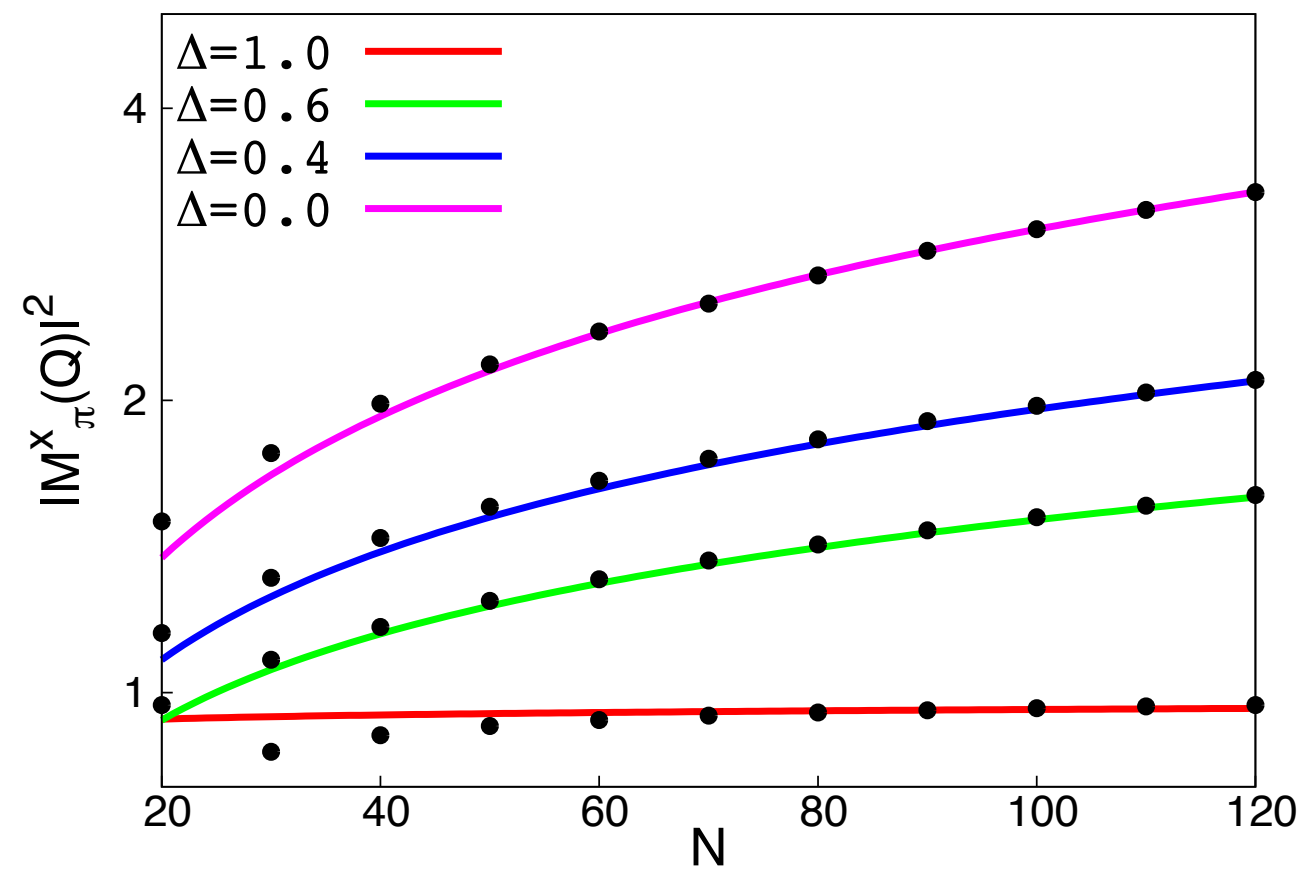

FIG. 20: $\left|\left\langle Q+\pi\left|S_{\pi}^{x}\right| Q\right\rangle\right|^{2}$ versus $N$ for $\Delta=1,0.6,0.4,0$ for $Q=2 \pi / 10$. The solid lines are the fitted curve of the form $\left|\left\langle Q+\pi\left|S_{\pi}^{x}\right| Q\right\rangle\right|^{2} \simeq 1 / N^{\frac{1}{2 \mathcal{Z}^{2}}-1}$ while the dots are the numerical data. 


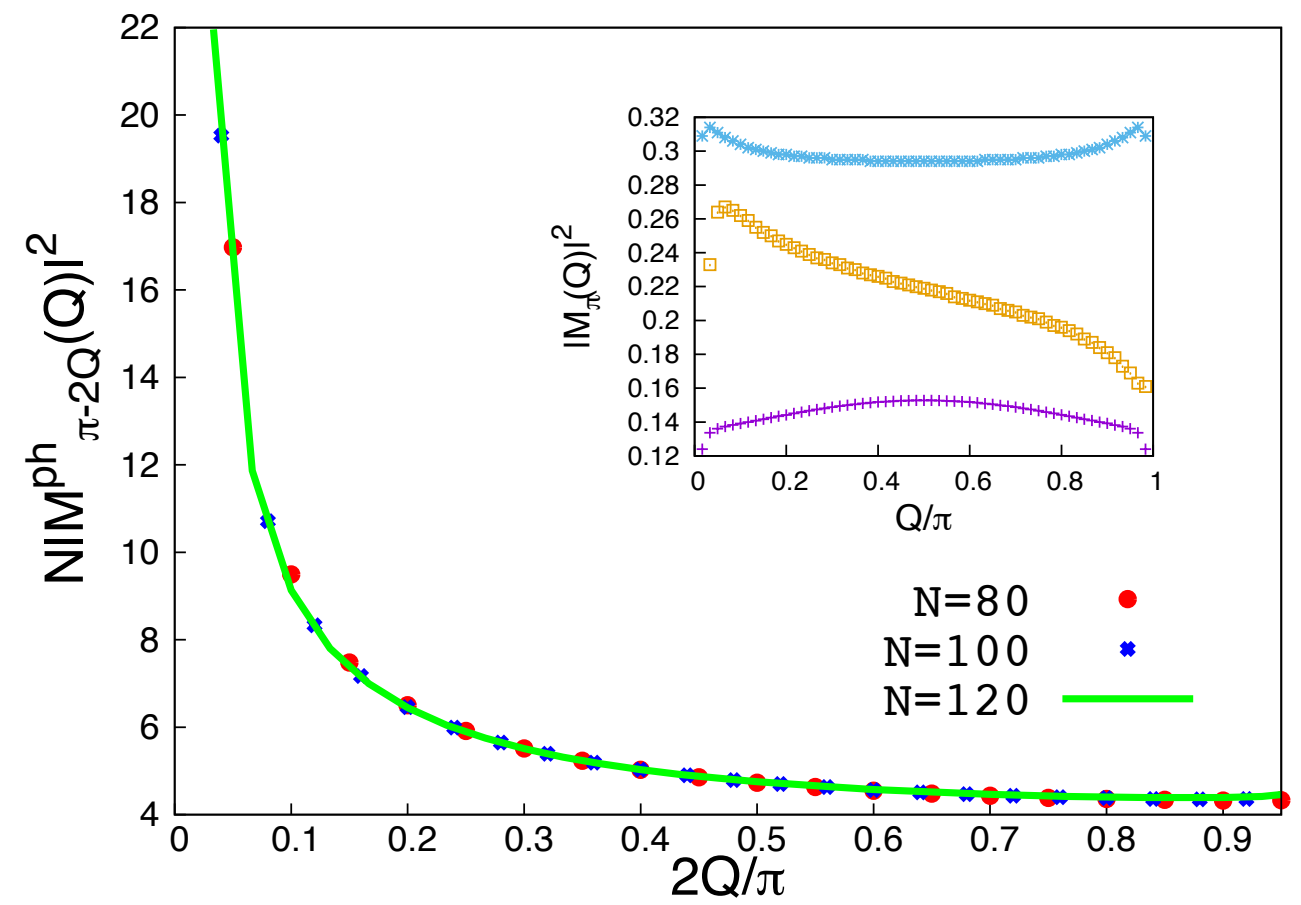

FIG. 21: $N\left|\left\langle\pi-Q\left|V_{\pi-2 Q}\right| Q\right\rangle\right|^{2}$ versus $Q$ for $\Delta=1$. The inset shows the $\pi$-transition versus $Q$ for the longitudinal $\left|M_{\pi}^{z}(Q)\right|^{2}$ (light blue), transverse $\left|M_{\pi}^{x}(Q)\right|^{2}$ (orange) and spin-phonon interaction $\left|M_{\pi}^{p h}(Q)\right|^{2}$ (purple) for $N=120$ and $\Delta=1$.

$G_{0}$ is the Green's function defined as

$$
G_{0}(E)=\lim _{\epsilon \rightarrow 0} \frac{1}{E-H_{0}+i \epsilon}=\lim _{\epsilon \rightarrow 0} \sum_{\{\lambda\}} \frac{|\{\lambda\}\rangle\langle\{\lambda\}|}{E-E_{\{\lambda\}}+i \epsilon}
$$

and $|\{\lambda\}\rangle$ denotes the Hamiltonian $H_{0}$ eigenstate parametrized by the set of parameters $\{\lambda\}$.

Next, we define the T-matrix, a "black box" that contains all the relevant information about the scattering process

$$
T=V\left(1-G_{0} V\right)^{-1}=V \sum_{k=0}^{\infty}\left(G_{0} V\right)^{k},
$$

from which we obtain the "diagonal" transmission probability (probability to find the particle in the same state) of a particle in the state $|Q\rangle$ with energy $\varepsilon_{Q}$ and group velocity $u_{Q}=d \varepsilon_{Q} / d Q[37]$

$$
\mathcal{T}_{Q, Q}=\left(1+\frac{N}{u_{Q}} \Im\langle Q|T| Q\rangle\right)^{2}+\left(\frac{N}{u_{Q}} \Re\langle Q|T| Q\rangle\right)^{2} .
$$

If we apply this formalism to the case of the anisotropic Heisenberg chain we obtain the Green's function for the single spinon,

$$
G_{0}(Q)=\lim _{\epsilon \rightarrow 0} \sum_{\{\lambda\}} \frac{|\{\lambda\}\rangle\langle\{\lambda\}|}{\varepsilon_{Q}-E_{\{\lambda\}}+i \epsilon}, \quad \varepsilon_{Q}=\frac{\pi}{2} \frac{\sin \gamma}{\gamma}|\sin Q| .
$$

Moreover, in the case of an even spin chain, since it is impossible to physically distinguish between two spinon excitations that have the same energy and group velocity but live in different branches of the Cloiseaux-Pearson spectrum, we also define the "non-diagonal" transmission probability as

$$
\mathcal{T}_{Q, Q+\pi}=\left(\frac{N}{u_{Q}} \Im\langle Q+\pi|T| Q\rangle\right)^{2}+\left(\frac{N}{u_{Q}} \Re\langle Q+\pi|T| Q\rangle\right)^{2} .
$$


In the odd site case, at least in the lower branch approximation, this is not needed.

Let us analytically evaluate the transmission probability for a "free" spinon (including only one-spinon excitations) toy model in an odd site spin chain with a potential matrix

$$
V=\frac{g}{N^{\alpha}} \sum_{Q, Q^{\prime}}|Q\rangle\left\langle Q^{\prime}\right|
$$

where $\alpha>0$ and $|Q\rangle,\left|Q^{\prime}\right\rangle$ are one-spinon states. Note that $\alpha=1$ corresponds to the case of a $\delta$-like potential which we presented in the main text. Using eqs.(29-32) we evaluate the $T$ matrix and the transmission amplitude $\mathcal{T}$, a function of $g_{e f f} / u_{Q}, g_{e f f} \equiv g N^{1-\alpha}$

$$
\begin{aligned}
T\left(Q, Q^{\prime}\right) & =\frac{g}{N^{\alpha}} \frac{1}{1-\left(I_{1}+i I_{2}\right)}, \\
I_{1} & =\frac{g_{\text {eff }}}{2 \pi} P \int \frac{d q}{\varepsilon_{Q}-\varepsilon_{q}}=-\frac{g_{\text {eff }}}{2 \pi\left|u_{Q}\right|} \log \left(\frac{1+|\cos Q|}{1-|\cos Q|}\right), \quad I_{2}=-\frac{g_{\text {eff }}}{\left|u_{Q}\right|}, \\
\mathcal{T}_{Q, Q} & =t_{1}^{2}+\left(1+t_{2}\right)^{2}, \\
t_{1} & =\left(\frac{g_{\text {eff }}}{u_{Q}}\right) \frac{\left(1-I_{1}\right)}{\left(1-I_{1}\right)^{2}+\left(I_{2}\right)^{2}}, \quad t_{2}=\left(\frac{g_{\text {eff }}}{u_{Q}}\right) \frac{I_{2}}{\left(1-I_{1}\right)^{2}+\left(I_{2}\right)^{2}} .
\end{aligned}
$$

This result holds for $I_{1}^{2}+I_{2}^{2}<1$. An interesting observation is that if we include only the on-shell matrix elements, i.e. only the $I_{2}$ part, then we get the correct qualitative behavior in the dependence on $N$ and the spinon energy $\varepsilon_{Q}$, with the rest of the matrix elements given by $I_{1}$ acting as corrections to the amplitude. Moreover, by repeating the same calculation for an even site spin chain, we can technically understand our results of the spinon transfer between the two branches, since for $\alpha<1$ the transmission probability $\mathcal{T}_{Q, Q} \rightarrow 0$ as $N$ increases while in the case $\alpha>1$ the transmission probability $\mathcal{T}_{Q, Q} \rightarrow 1$ as $N$ increases and $\mathcal{T}_{Q, Q}$ is scale invariant when $\alpha=1$.

Furthermore, using only the dominant matrix elements we can obtain a qualitative expression for the transmission amplitude of the potentials that we have previously discussed. Also we use the symmetry with respect to $Q= \pm \pi / 2$, which holds for the longitudinal and the spin-phonon interaction, while for the transverse potential we notice that although this condition is not fulfilled, the same procedure gives essentially the correct result. Let us first apply the above for an even site spin chain.

The diagonal element of the T-matrix is given by

$$
\begin{aligned}
T(Q, Q) & =i \frac{u_{Q}}{N} \sum_{n=1}^{\infty}(-1)^{n}\left(\frac{1}{2 u_{Q}}\right)^{2 n}\left(\mathcal{F}^{n}+\mathcal{G}^{n}\right), \\
\mathcal{F} & \equiv N\left(\left|\left\langle Q+\pi\left|V_{\pi}\right| Q\right\rangle\right|+\left|\left\langle\pi-Q\left|V_{\pi-2 Q}\right| Q\right\rangle\right|\right)^{2} \\
\mathcal{G} & \equiv N\left(\left|\left\langle Q+\pi\left|V_{\pi}\right| Q\right\rangle\right|-\left|\left\langle\pi-Q\left|V_{\pi-2 Q}\right| Q\right\rangle\right|\right)^{2},
\end{aligned}
$$

where we have used that only the even terms contribute to the Born series and that

$$
\sum_{m=e v e n}^{2 n}\left(\begin{array}{c}
2 n \\
m
\end{array}\right)\left|\left\langle Q+\pi\left|V_{\pi}\right| Q\right\rangle\right|^{2 n-m}\left|\left\langle\pi-Q\left|V_{\pi-2 Q}\right| Q\right\rangle\right|^{m}=\frac{1}{2 N^{n}}\left(\mathcal{F}^{n}+\mathcal{G}^{n}\right) .
$$

Summing up the series to obtain the diagonal T-matrix element,

$$
T(Q, Q)=-i \frac{u_{Q}}{N}\left[\frac{\mathcal{F}}{4 u_{Q}^{2}+\mathcal{F}}+\frac{\mathcal{G}}{4 u_{Q}^{2}+\mathcal{G}}\right]
$$

and the transmission amplitude,

$$
\mathcal{T}_{Q, Q}=\left(\frac{16 u_{Q}^{4}-\mathcal{F G}}{16 u_{Q}^{4}+\mathcal{F G}+4 u_{Q}^{2}(\mathcal{F}+\mathcal{G})}\right)^{2} .
$$

Additionally we calculate the probability that the spinon is transmitted through the second branch. Using a similar procedure we obtain the $T(Q, Q+\pi)$ T-matrix element

$$
\begin{aligned}
T(Q, Q+\pi)= & 2 u_{Q}^{2}\left[\frac{\left|\left\langle Q+\pi\left|V_{\pi}\right| Q\right\rangle\right|+\left|\left\langle\pi-Q\left|V_{\pi-2 Q}\right| Q\right\rangle\right|}{4 u_{Q}^{2}+\mathcal{F}}\right. \\
& \left.+\frac{\left|\left\langle Q+\pi\left|V_{\pi}\right| Q\right\rangle\right|-\left|\left\langle\pi-Q\left|V_{\pi-2 Q}\right| Q\right\rangle\right|}{4 u_{Q}^{2}+\mathcal{G}}\right],
\end{aligned}
$$


and $\mathcal{T}_{Q, Q+\pi}=\left(\frac{N}{u_{Q}}\right)^{2} T(Q, Q+\pi)^{2}$.

In the case of an odd site spin chain we have a much simpler situation, since the $\pi$-transfer matrix element and the second branch are non-existent.

Finally note that, when $\varepsilon_{Q}$ is not close to zero, the quantity $N\left\langle\pi-Q\left|S_{\pi-2 Q}^{z}\right| Q\right\rangle=f^{z}(Q)$ can be considered negligible. Therefore by taking the logarithm of the transmission coefficient and using the identity $\log (1-x)=x-x^{2} / 2+O\left(x^{3}\right)$, we obtain that for $\left(g_{e f f} / u_{Q}\right)^{2} / 4 f^{z}(Q)<<1$,

$$
\log \mathcal{T}_{Q, Q} \simeq-f^{z}(Q)\left(\frac{g_{\text {eff }}}{u_{Q}}\right)^{2} \Rightarrow \mathcal{T}_{Q, Q} \simeq e^{-f^{z}(Q)\left(\frac{g_{e f f}}{u_{Q}}\right)^{2}}, \quad g_{\text {eff }} \equiv g N^{1-\mathcal{Z}^{2}} .
$$

